\title{
An efficient two-stage Markov Chain Monte Carlo method for dynamic data integration
}

\author{
Y. Efendiev, ${ }^{1}$ A. Datta-Gupta, ${ }^{2}$ V. Ginting, ${ }^{1}$ X. $\mathrm{Ma}^{2},{ }^{2}$ and B. Mallick ${ }^{3}$
}

\section{Introduction}

With increasing interest in accurate prediction of subsurface properties, subsurface characterization based on dynamic data such as transient pressure, tracer and multiphase production response (phase fractional flow), takes on greater importance. Uncertainties on the detailed description of subsurface porosity and permeability are large contributors to the overall uncertainty in performance forecasting. Reducing this uncertainty can be achieved by integrating additional data in subsurface modeling. Integration of data from different sources is a nontrivial task because different data sources scan different length scales of heterogeneity and can have different degrees of precision. For example, well logs can resolve heterogeneity at the scale of a few feet whereas fractional flow data, which is the ratio of the injection fluid to the total fluid produced, usually scan the length scales comparable to the inter-well distances. In particular, integration of dynamic data leads to an inverse problem. Such inverse problems are computationally expensive and typically requires orders of magnitude more computation time compared to the forward simulation of flow and transport. Several authors have proposed techniques to circumvent these problems [Rubin and Dagan, 1987; Carrera and Neuman, 1986; Andeman and Hill, 1999].

Because flow and transport in porous media are nonlinear, it is generally difficult to calculate directly the probability distribution for permeability fields conditioned to dynamic data. Instead, we are forced to estimate the probability distributions from the outcomes of flow predictions for a large number of realizations. For this method to work, it is essential that these permeability realizations adequately reflect the uncertainty in subsurface properties. The type of the problem that we consider is one in which the permeability in a large number of gridcells must be determined for high resolution subsurface flow and transport calculations and predictions. Our data sources will consist of both static data such as cores/well logs and dynamic data such as water-cut data at the producing wells. In addition, we assume that a reasonable prior covariance of the permeability field can be derived based on static data, and we specify full prior distribution. Markov chain Monte Carlo methods are well suited for reconstructing the spatial distribution of permeability and quantifying the associated uncertainties, and they are used in petroleum applications (see e.g., Baker et al. [2001]). General framework of MCMC allows one to incorporate complicated relations between observed data (fractional flow data) and permeability field and does not require the normalizing constant of probability distributions which is difficult to compute. On the other hand one of the main disadvantages of MCMC is its computational cost. Each proposal of MCMC requires the solution of the forward problem. Thus, it is important to devise MCMC algorithms which have high acceptance rate.

The goal of this paper is to employ coarse-scale models based on single-phase upscaling to increase the acceptance rate of Markov chain Monte Carlo approaches for subsurface characterization. The main idea of this upscaling approach, is that only the absolute permeability field is upscaled, and the system of equations is solved on the coarse-grid. The sampling of permeability field based on the water-cut data is, in general, very expensive because each proposal requires a fine-scale simulation. Previously coarse-scale and approximate models have been used to reduce the computational cost [Omre and Lodoen, 2004; Lodoen et al. 2004]. One of the first paper, where the use of coarse-scale models is promoted, is Glimm and Sharp [1998]. These approaches are based on the estimation of upscaling errors. In our paper, we do not use coarse-scale models as an approximation for the fine-scale models. The coarse-scale computations based on single-phase upscaling are used to decide whether to run the fine-scale simulation in order to determine the acceptance for a given proposal during MCMC. The latter introduces two-stage MCMC approach and modifies the underlying Markov chain which has been taken into account in an appropriate way in our paper. The proposed approach analyzed rigorously in Efendiev et al. [2005s], where we use a different type of upscaled model. Two-stage MCMC approaches have been used in statistical literature before (e.g., Christen and Fox, 2005). The idea of developing approaches with the aim of increasing the acceptance rate in MCMC simulations is not new. Kitanidis, [1995] and Oliver et al. [1996, 1997] used geostatistical inverse methods for proposing permeability fields that yield high acceptance rate using gradient based techniques. These approaches are different from the approach presented in this paper. We do not employ gradient-based inverse methods, but rather use coarse-scale simulations to pre-determine whether or not to do fine-scale simulations. The results of the fine-scale simulation are then used in conjunction with Metropolis-Hasting criteria to update permeability field during subsurface characterization.

For our numerical results, we consider a five-spot problem, where the injection well is placed in the middle of the square domain and four production wells are placed at the vertices of the square. The permeability field is 
assumed to be log-normal and known at the well locations. Using single-phase upscaling in two-stage MCMC, we achieve several fold increase in the acceptance rate.

\section{Fine- and Coarse-Scale Models}

In this section we briefly introduce the coarse-scale model used in the simulations. We consider two-phase flow in a subsurface formation (denoted by $\Omega$ ) under the assumption that the displacement is dominated by viscous effects. For clarity of exposition, we neglect the effects of gravity, compressibility, and capillary pressure, although our proposed approach is independent of the choice of physical mechanisms. Also, porosity will be considered to be constant. The two phases will be referred to as water and oil (or a non-aqueous phase liquid), designated by subscripts $w$ and $o$, respectively. We write Darcy's law for each phase as follows:

$$
v_{j}=-\frac{k_{r j}(S)}{\mu_{j}} k \nabla p
$$

where $v_{j}$ is the phase velocity, $k$ is the permeability tensor, $k_{r j}$ is the relative permeability to phase $j(j=o, w)$, $S$ is the water saturation (volume fraction) and $p$ is the pressure. In this work, a single set of relative permeability curves is used and $k$ is taken to be a diagonal tensor. Combining Darcy's law with a statement of conservation of mass allows us to express the governing equations in terms of pressure and saturation equations:

$$
\begin{aligned}
& \nabla \cdot(\lambda(S) k \nabla p)=Q_{s}, \\
& \frac{\partial S}{\partial t}+v \cdot \nabla f(S)=0,
\end{aligned}
$$

where $\lambda$ is the total mobility, $Q_{s}$ is source term, $f$ is the fractional flux of water, and $v$ is the total velocity, which are respectively given by:

$$
\begin{gathered}
\lambda(S)=\frac{k_{r w}(S)}{\mu_{w}}+\frac{k_{r o}(S)}{\mu_{o}}, \\
f(S)=\frac{k_{r w}(S) / \mu_{w}}{k_{r w}(S) / \mu_{w}+k_{r o}(S) / \mu_{o}}, \\
v=v_{w}+v_{o}=-\lambda(S) k \cdot \nabla p .
\end{gathered}
$$

The above descriptions are referred to as the fine-scale model of the two-phase flow problem.

Next, we will briefly describe single-phase flow upscaling procedure for two-phase flow in heterogeneous porous media. This type of approaches for upscaling are discussed by many authors; see e.g., Christie[1996]; Durlofsky [1998]. The main idea of this approach is to upscale the absolute permeability field $k$ on the coarse-grid (see Figure 1), then solve the original system on the coarse-grid with upscaled permeability field. Below, we will discuss briefly the upscaling of absolute permeability used in our simulations.

Consider the fine-scale permeability that is defined in the domain with underlying fine grid as shown in Figure 1. On the same graph we illustrate a coarse-scale partition of the domain. To calculate the upscaled permeability field at the coarse-level, we use the solutions of local pressure equations. The main idea of the calculation of a coarse-scale permeability is that it delivers the same average response as that of the underlying fine-scale problem locally. For each coarse domain $K$, we solve the local problems

$$
\operatorname{div}\left(k(x) \nabla \phi_{j}\right)=0,
$$

with some coarse-scale boundary conditions. Here $k(x)$ denotes the fine-scale permeability field. We will use the boundary conditions which are given by $\phi_{j}=1$ and $\phi_{j}=0$ on the opposite sides along the direction $e_{j}$ and no flow boundary conditions on all other sides. For these boundary conditions, the coarse-scale permeability tensor is given by

$$
\left(k^{*}(x) e_{j}, e_{l}\right)=\frac{1}{|K|} \int_{K}\left(k(x) \nabla \phi_{j}(x), e_{l}\right) d x
$$

where $\phi_{j}$ is the solution of (7) with prescribed boundary conditions. Various boundary condition can have some influence on the accuracy of the calculations, including periodic, Dirichlet and etc. These issues have been discussed in $W u$ et al. [2002]. In particular, for determining the coarse-scale permeability field one can choose the local domains that are larger than the target coarse block, $K$, for (7).

Once the upscaled absolute permeability is computed, the original equation is solved on the coarse-grid, without changing the form of relative permeability curves. This is an inexpensive calculations, since the pressure update 
involves only solving the pressure equation on the coarse-grid, and one can take larger time step for solving the transport equation. In our numerical simulations, the fine-grid is coarsened 10 times in each direction. This provides 100 times speed-up for each pressure update. As a result, the upscaling of two-phase flow based on absolute permeability upscaling provides more than 100 times speed-up. For solving the saturation equation, we employ streamline approach to avoid the numerical diffusion. Note that the upscaling of the saturation equation does not take into account subgrid effects. These kinds of upscaling techniques in conjunction with the upscaling of absolute permeability has been used in groundwater applications (see e.g. Durlofsky et al. [1997]).

Our goal in this paper, is to sample fine-scale permeability field based on phase fractional flow, specifically, the fraction of water produced in relation to the total production rate. For two-phase water-oil flow, the fractional flow or water-cut $F(t)$ (denoted simply by $F$ in further discussion) is defined as the fraction of water in the produced fluid and is given by $q_{w} / q_{t}$, where $q_{t}=q_{o}+q_{w}$, with $q_{o}$ and $q_{w}$ the flow rates of oil and water at the production edge of the model,

$$
F(t)=\frac{\int_{\partial \Omega^{\text {out }}} v_{n} f(S) d l}{\int_{\partial \Omega^{\text {out }}} v_{n} d l},
$$

where $\partial \Omega^{\text {out }}$ is outflow boundaries and $v_{n}$ is normal velocity field. Typically, the coarse- and fine-scale water-cut curves can be quite different. However, within the sampling approach, the strong correlation between fine- and coarse-scale water-cut curves plays an important role.

\section{Markov Chain Monte Carlo (MCMC) method using coarse-scale models}

To find the permeability field given water-cut information, we assume that an observed water-cut data, $F^{r e f}(t)$, is given. Consequently, one can consider this problem as a sampling from the conditional distribution $P\left(k \mid F^{r e f}\right)$. Using Bayes theorem we can write

$$
P\left(k \mid F^{r e f}\right) \propto P\left(F^{r e f} \mid k\right) P(k) .
$$

The normalizing constant in this expression is not important, because we use iterative updating procedure. In (10), $P\left(F^{r e f} \mid k\right)$ represents the likelihood function and requires the forward solution of flow and transport. We will be using Metropolis-Hasting MCMC (see Robert and Casella, 1999) to sample from the posterior distribution $P(k \mid F)$. The main idea of MCMC is to generate a Markov chain whose stationary distribution is given by $P\left(k \mid F^{r e f}\right)$. At each iteration, a permeability field, $k$, is proposed using instrumental distribution $q\left(k \mid k_{n}\right)$ (where $k_{n}$ is previously accepted permeability field), and then forward problem is solved to determine the acceptance probability,

$$
\operatorname{Pr}\left(k_{n}, k\right)=\min \left(1, \frac{q\left(k_{n} \mid k\right) P\left(k \mid F^{r e f}\right)}{q\left(k \mid k_{n}\right) P\left(k_{n} \mid F^{r e f}\right)}\right),
$$

i.e. $k_{n+1}=k$ with probability $\operatorname{Pr}\left(k_{n}, k\right)$, and $k_{n+1}=k_{n}$ with probability $1-\operatorname{Pr}\left(k_{n}, k\right)$.

Since each proposal requires the fine-scale computation, direct (full) MCMC is expensive. Typically, direct MCMC requires several thousand iterations for the convergence to a steady state, where each iteration involves the computation of the fine-scale solution over a large time interval. One way to achieve efficiency is to propose an algorithm that increases the acceptance rate of MCMC. This minimizes rejection of proposals after detailed flow and transport calculations. In this paper, we use coarse-scale solutions based on single-phase upscaling to increase the acceptance rate. The main idea of this algorithm is to compare the fractional flow curves that correspond to the coarse-scale models to determine whether or not to run fine-scale simulations.

Algorithm (coarse-scale MCMC)

- Step 1. At $k_{n}$ generate $k$ from $q\left(k \mid k_{n}\right)$.

- Step 2. Accept $k$ for the fine-scale run with probability

$$
G\left(k_{n}, k\right)=\min \left(1, \frac{q\left(k_{n} \mid k\right) P\left(k^{*} \mid F^{r e f}\right)}{q\left(k \mid k_{n}\right) P\left(k_{n}^{*} \mid F^{r e f}\right)}\right),
$$

i.e. $k_{n+1}=k$ (conditionally) with probability $G\left(k_{n}, k\right)$, and $k_{n+1}=k_{n}$ (conditionally) with probability $1-$ $G\left(k_{n}, k\right)$. If rejected go to step 1 .

- Step 3. Accept $k$ with probability

$$
\operatorname{Pr}\left(k_{n}, k\right)=\min \left(1, \frac{Q\left(k_{n} \mid k\right) P\left(k \mid F^{r e f}\right)}{Q\left(k \mid k_{n}\right) P\left(k_{n} \mid F^{r e f}\right)}\right),
$$

i.e. $k_{n+1}=k$ with probability $\operatorname{Pr}\left(k_{n}, k\right)$, and $k_{n+1}=k_{n}$ with probability $1-\operatorname{Pr}\left(k_{n}, k\right)$. The proposal function $Q\left(k \mid k_{n}\right)$ satisfies

$$
Q\left(k \mid k_{n}\right)=G\left(k_{n}, k\right) q\left(k \mid k_{n}\right)+\left(1-\int G\left(k_{n}, k\right) q\left(k \mid k_{n}\right) d k\right) \delta_{k_{n}}(k) .
$$


First we note that $Q$ is the transition distribution for the modified Markov chain, which simply indicates that the chain moves from $k_{n}$ to $k$ with probability $G\left(k_{n}, k\right) q\left(k \mid k_{n}\right)$ and remains at $k_{n}$ with the probability $1-Q\left(k \mid k_{n}\right)$. Moreover, there is no need to compute $\left(1-\int G\left(k_{n}, k\right) q\left(k \mid k_{n}\right) d k\right) \delta_{k_{n}}(k)$ during the simulations. Indeed, if $k=k_{n}$ the chain remains at the same point, otherwise $k \neq k_{n}, Q\left(k \mid k_{n}\right)=G\left(k_{n}, k\right) q\left(k \mid k_{n}\right)$. In Efendiev et al. [2005s], we have shown that $\operatorname{Pr}\left(k_{n}, k\right)$ can be easily computed by

$$
\operatorname{Pr}\left(k_{n}, k\right)=\min \left(1, \frac{P\left(k \mid F^{\text {ref }}\right) P\left(k_{n}^{*} \mid F^{\text {ref }}\right)}{P\left(k_{n} \mid F^{r e f}\right) P\left(k^{*} \mid F^{r e f}\right)}\right) .
$$

For our numerical results, we choose

$$
P\left(F^{r e f} \mid k\right) \propto \exp \left(-\left(F^{r e f}-F_{k}\right) \Sigma\left(F^{r e f}-F_{k}\right)\right)
$$

where $F^{r e f}$ is the reference water-cut curve, $F_{k}$ is the water-cut curve that is obtained from the simulations with permeability $k$, and $\Sigma$ is covariance matrix representing the measurement errors. Water-cut depends on time and measured at some time instants (i.e., $F^{r e f}-F_{k}$ is a finite dimensional vector and $\Sigma$ is square matrix). Since only the ratio is involved in the acceptance criteria the normalization constant is not important. We denote the covariance matrix for fine-scale simulations by $\Sigma_{f}$. For coarse-scale simulations, we choose the covariance matrix to be $\Sigma_{c}$,

$$
P\left(F^{r e f} \mid k^{*}\right) \propto \exp \left(-\left(F^{r e f}-F_{k^{*}}\right) \Sigma_{c}\left(F^{r e f}-F_{k^{*}}\right)\right)
$$

In our numerical results, we take $\Sigma_{f}=\sigma_{f} I$ and $\Sigma_{c}=\sigma_{c} I$, where $I$ is identity matrix. First, we would like to note that the Gaussian error model for the coarse-scale model is not justified, and we only use it in our second step in MCMC to decide whether or not to run the fine-scale simulations. The choice of the parameter $\sigma_{c}$ is crucial for increasing the acceptance rate. We have tested various choices of these parameters in our simulations. Larger $\sigma_{c}$ corresponds to the full MCMC where all the proposals are tested using fine-scale simulations. Numerical results show that the optimal $\sigma_{c}$ is approximately equal to $\sigma_{f}$, and that the two-stage MCMC approach improves the acceptance rate. This is due to the fact that the correlation between fine- and coarse-scale water-cut data is strong. In particular, we have found that the correlation between $\left(F^{r e f}-F_{k}\right) \Sigma_{f}\left(F^{r e f}-F_{k}\right)$ and $\left(F^{r e f}-F_{k^{*}}\right) \Sigma_{c}\left(F^{r e f}-F_{k^{*}}\right)$ to be 0.9 for the numerical examples, we have studied. We have analyzed this approach rigorously in Efendiev at al. [2005s], where we use different type of upscaling method. In particular, we showed that the modified chain is ergodic and converges to the correct steady state distribution.

\section{Numerical Results}

In this section, we present a set of representative simulation results for two-phase flow. We consider two dimensional system in a square domain $\Omega$ with unit size. The method can be naturally extended to three dimensional case. We set the relative permeabilities of oil and water to be simple quadratic functions of their respective saturations; i.e., $k_{r w}=S^{2}$ and $k_{r o}=(1-S)^{2}$, where $S$ is the water saturation. We set the ratio of oil to water viscosity $\left(M=\mu_{o} / \mu_{w}\right)$ to be 5 . We consider five-spot problem, where the injection well is placed at the middle of the square domain and four production wells are placed at the vertices.

The fine-scale models are of dimension $60 \times 60$ and the coarse-scale models, generated through a uniform coarsening of the fine model, are $6 \times 6$. As for prior distribution, we assume the permeability is log-normal, $\log (k(x))=Y(x)$, where $Y(x)$ has Gaussian distribution with correlation function

$$
R\left(x_{1}, z_{1} ; x_{2}, z_{2}\right)=\sigma^{2} \exp \left(-\frac{\left|x_{1}-x_{2}\right|^{2}}{2 l_{x}^{2}}-\frac{\left|z_{1}-z_{2}\right|^{2}}{2 l_{z}^{2}}\right)
$$

with correlation lengths $l_{x}=l_{z}=0.2$ and variance $\sigma^{2}=2$. Using the Karhunen-Loeve expansion [Wong 1971], the permeability field can be expanded in terms orthogonal basis $\phi_{k}(x, z)$ satisfying

$$
\int_{\Omega} R\left(x_{1}, z_{1} ; x_{2}, z_{2}\right) \phi_{k}\left(x_{2}, z_{2}\right) d x_{2} d z_{2}=\lambda_{k} \phi_{k}\left(x_{1}, z_{1}\right), \quad k=1,2, \ldots,
$$

where $\lambda_{k}=E\left[Y_{k}^{2}\right]>0$. Consequently, the permeability field can be written as

$$
Y(x, \omega)=\sum_{k=1}^{\infty} \sqrt{\lambda_{k}} \theta_{k}(\omega) \phi_{k}(x)
$$

This is a well known technique and is called Karhunen-Loeve expansion. Next, we identify the dominant eigenvalues, which gives less than 5 percent errors for the solution if only dominant eigenvalues are used. For the 
permeability field considered in our numerical example, 20 eigenvalues are sufficient. Consequently, the dimension of the parameter space is 20 . This is a significant dimension reduction, since originally the permeability field has $60 \times 60=3600$ dimension. Furthermore, we assume that the values of the log-permeability at the well locations are known. This condition is imposed by setting

$$
\sum_{k=1}^{20} \sqrt{\lambda_{k}} \theta_{k} \phi_{k}\left(x_{j}\right)=\alpha_{j},
$$

where $\alpha_{j}(j=1, \ldots, 5)$ are prescribed constants, and $x_{j}$ are well locations. For simplicity, we set $\alpha_{j}=0$ for all $j=1, \ldots, 5$. In the simulations we propose fifteen $\theta_{i}$ and calculate the rest of $\theta_{i}$ by solving the linear system (21).

As for the proposal distribution, we choose random walk sampler, where $q(x \mid y)=y+\delta * \epsilon$, where $\epsilon$ is Gaussian random variable with zero mean and variance 1 . In our numerical simulations, we choose $\delta=0.3$ and $\sigma_{f}=1$. In Figure 2, we plot the acceptance rate for 5 different values of $\sigma_{c}$, where we specify only the ratio between $\sigma_{c}$ and $\sigma_{f}$. Here the acceptance rate denotes the number of accepted fine-scale permeability field divided by total number of fine-scale simulations. As we see from this figure that, the use of the coarse-scale models increases the acceptance rate several times. In particular, if $\sigma_{c}$ is larger, more proposals pass the second step, and thus the acceptance rate of modified MCMC is reduced. In the limit of very large $\sigma_{c}$, the acceptance rate of modified MCMC approaches to that of direct MCMC. For smaller $\sigma_{c}, \sigma_{c}=2 \sigma_{f}$, we observe 8 times increase in the acceptance rate. In the next figure, Figure 3, we plot the cumulative water-cut errors as a function of iterations for both direct and modified MCMC. Here, cumulative water-cut error denotes the sum of the water-cut errors of four production wells. We observe from this figure that both methods reach the steady state within 100 iterations, i.e., after 100 proposals. In Figure 4, water-cut curves at production wells for initial guess and one of the final sampled realizations are shown. Each figure represent a water-cut in one of the production wells. As we see, the sampled realization, captures correctly the observed water-cut. However, the realizations of the permeability field are not necessarily very close to the reference permeability field, as one can see from Figure 5.

We have tested two-stage MCMC approach using other type, simpler, prior distributions. In particular, we have considered the prior distribution to be linear combination of given permeability fields sampled from a distribution (similar to gradual deformation method [Hu 2000]). For this type of problems, we have observed an order of magnitude increase in the acceptance rate for both independent and random walk samplers. Moreover, for this type of prior, we have observed very good agreement between the sampled permeability fields and the reference permeability field. We do not present these numerical results here. Finally, we would like to discuss the CPU savings achieved using two-stage MCMC with single-phase upscaling. In the proposed approach, the model is coarsened 100 times (10 times in each direction). As we mentioned before, this provides 100 times speed-up for each coarse-scale run. If we consider the case of very large $\sigma_{c}$ (i.e., no increase in the acceptance rate), the CPU time for two-stage MCMC is 1 percent higher compared to the direct MCMC. However, in general, if the acceptance rate is increased $n$ times by two-stage MCMC, then the speed-up is more than $n$-times. Consequently, in the numerical example studied in the paper, we have achieved 5-8 times speed-up using single-phase upscaling in two-stage MCMC method.

\section{Conclusion}

In the paper we use two-stage MCMC that utilizes inexpensive coarse-scale models to screen out detailed flow and transport simulations. The main idea of the method is to compare the proposed fields using coarse-scale methods prior to running the fine-scale simulations. If it is accepted (conditionally), then the fine-scale simulations are performed and it is decided using Metropolis-Hasting rule whether or not to accept the proposal. Because the differences between fine-scale fractional flows and corresponding coarse-scale fractional flows are correlated, we obtain several fold increase in acceptance rate.

\section{Acknowledgments}

We would like to acknowledge reviewers for valuable comments and suggestions. This work is supported by NSF grant DMS-0327713.

\section{References}

Andeman, E. and M. Hill (1999), A new multistage groundwater transport inverse method: Presentation, evaluation, and implications, Water Resour. Res., 35, 1053-1063.

Baker, J., M. Cuypers, and L. Holden (2001), Quantifying uncertainty in production forecasts: Another look at the PUNQ-S3 problem, SPE Journal, 6(4), 433-441.

J. Carrera and S. Neuman (1986), Estimation of aquifer parameters under transient and steady state conditions. 1. Maximum likelihood method for incorporating prior information, Water Resour. Res., 22, 199-210. 
Christen A. and C. Fox (2005), MCMC using an approximation, Journal of Computational and Graphical Statistics, to appear

Christie, M. (1996), Upscaling for reservoir simulation, J. Pet. Tech., 1004-1010.

Durlofsky, L.J. (1998), Coarse scale models of two phase flow in heterogeneous reservoirs: Volume averaged equations and their relationship to the existing upscaling techniques, Computational Geosciences, 2, 73-92.

Durlofsky, L.J., R. C. Jones, and W. J. Milliken (1997), A nonuniform coarsening approach for the scale up of displacement processes in heterogeneous media, Advances in Water Resources, 20, 335-347.

Glimm, J. and D. H. Sharp (1998), Prediction and the quantification of uncertainty, Phys. D, 133, 152-170.

Efendiev, Y., A. Datta-Gupta, I. Osaka, and B. Mallick (2005), Multiscale data integration using coarse-scale models, Advances in Water Resources, 28, 303-314.

Efendiev, Y., T. Hou and W. Luo (2005s), Preconditioning Markov chain Monte Carlo simulations using coarse-scale models, Submitted.

Hu, L. (2000), Gradual deformation and iterative calibration of Gaussian-related stochastic models, Mathematical Geology, 32(1), 87-108.

Kitanidis, P. (1995), Quasi-linear geostatistical theory for inversing, Water Resour. Res., 31, 2411-2419.

Lodoen, O., H. Omre, L. Durlofsky, and Y. Chen (2004), Assessment of uncertainty in reservoir production forecasts using upscaled models, Submitted

Oliver, D., L. Cunha, and A. Reynolds (1997), Markov chain Monte Carlo methods for conditioning a permeability field to pressure data, Mathematical Geology, 29

Oliver, D., N. He, and A. Reynolds (1996), Conditioning permeability fields to pressure data, 5th European conference on the mathematics of oil recovery, Leoben, Austria, 3-6 September

Omre, H. and O. Lodoen (2005), Improved production forecasts and history matching using approximate models, to appear in SPE Journal September.

Robert, C. and G. Casella (1999), Monte Carlo Statistical Methods, Springer-Verlag, New-York, 1999.

Rubin, Y., and G. Dagan (1987), Stochastic identification of transmissivity and effective recharge in steady groundwater flow. 2. Case study, Water Resour. Res., 23, 1193-1200.

Wong, E. (1971), Stochastic Processes in Information and Dynamical Systems, MCGraw-Hill, 1971.

Wu, X.H., Y. R. Efendiev, and T. Y. Hou (2002), Analysis of upscaling absolute permeability, Discrete and Continuous Dynamical Systems, Series B,2, 185-204.

Y. Efendiev, Institute for Scientific Computation and Department of Mathematics, Texas A\&M University, College Station, TX 77843

A. Datta-Gupta, Department of Petroleum Engineering, Texas A\&M University, College Station, TX 77843

V. Ginting, Institute for Scientific Computation and Department of Mathematics, Texas A\&M University, College Station, TX 77843

X.Ma, Department of Petroleum Engineering, Texas A\&M University, College Station, TX 77843

B. Mallick, Department of Statistics, Texas A\&M University, College Station, TX 77843 


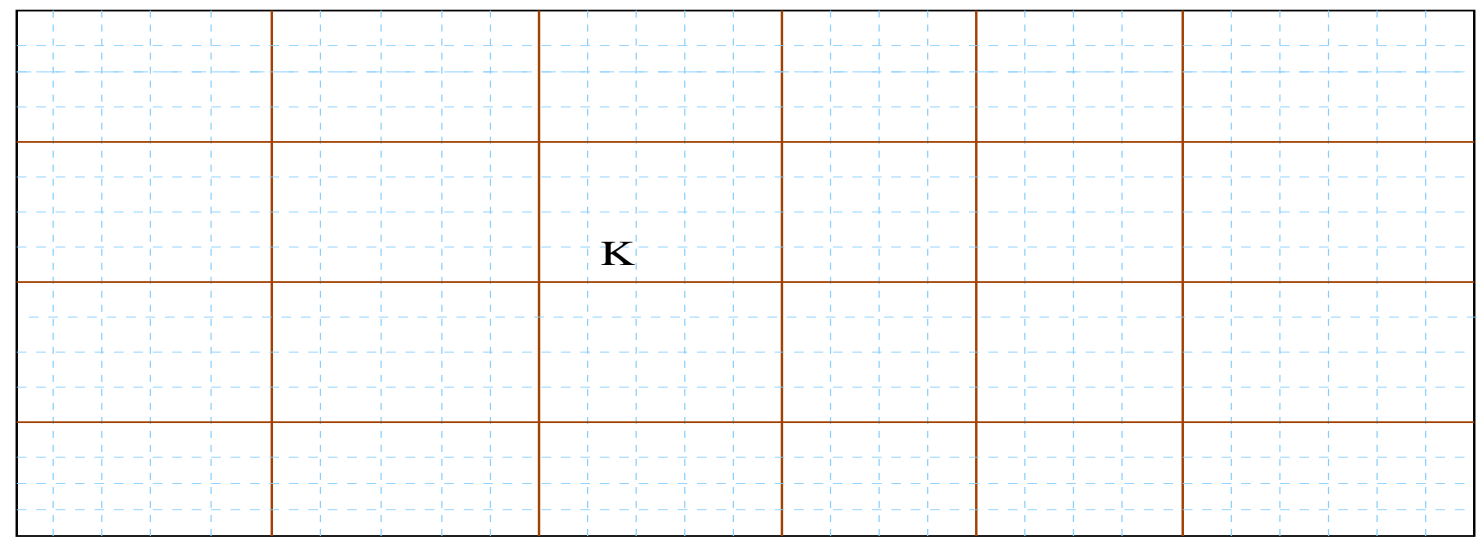

Coarse-grid

Fine-grid

Figure 1. Schematic description of fine- and coarsegrids. Bold lines illustrate a coarse-scale partitioning, while thin lines show a fine-scale partitioning within coarse-grid cells.

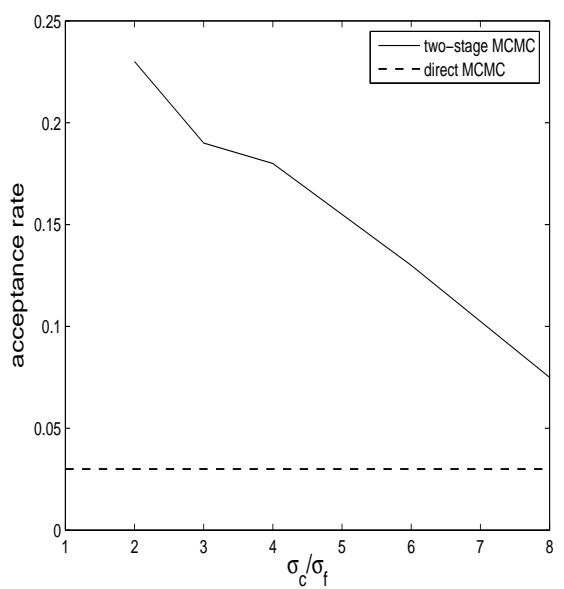

Figure 2. Acceptance rate for modified and direct MCMC methods. 

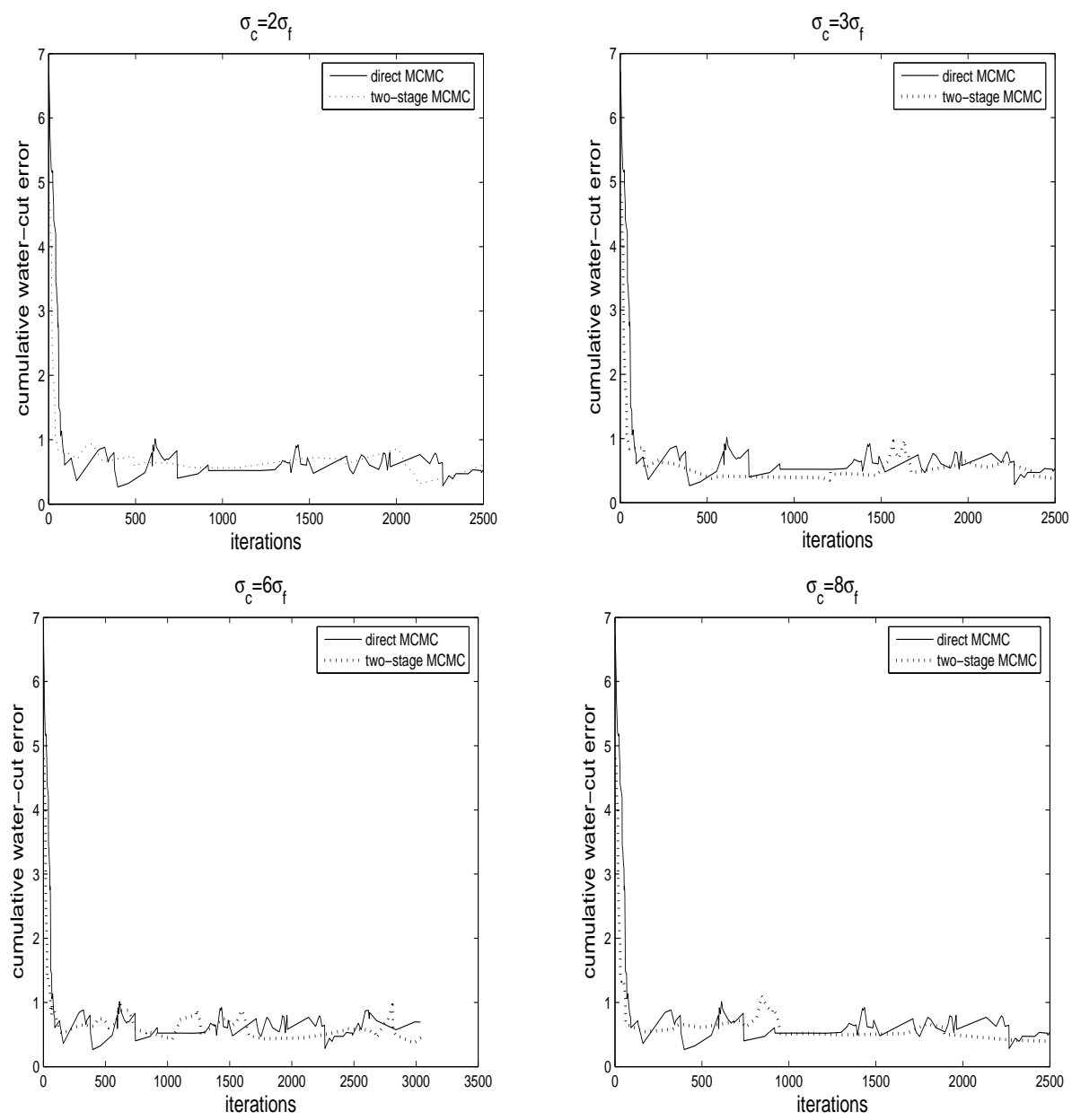

Figure 3. Cumulative water-cut error as a function of iterations. 

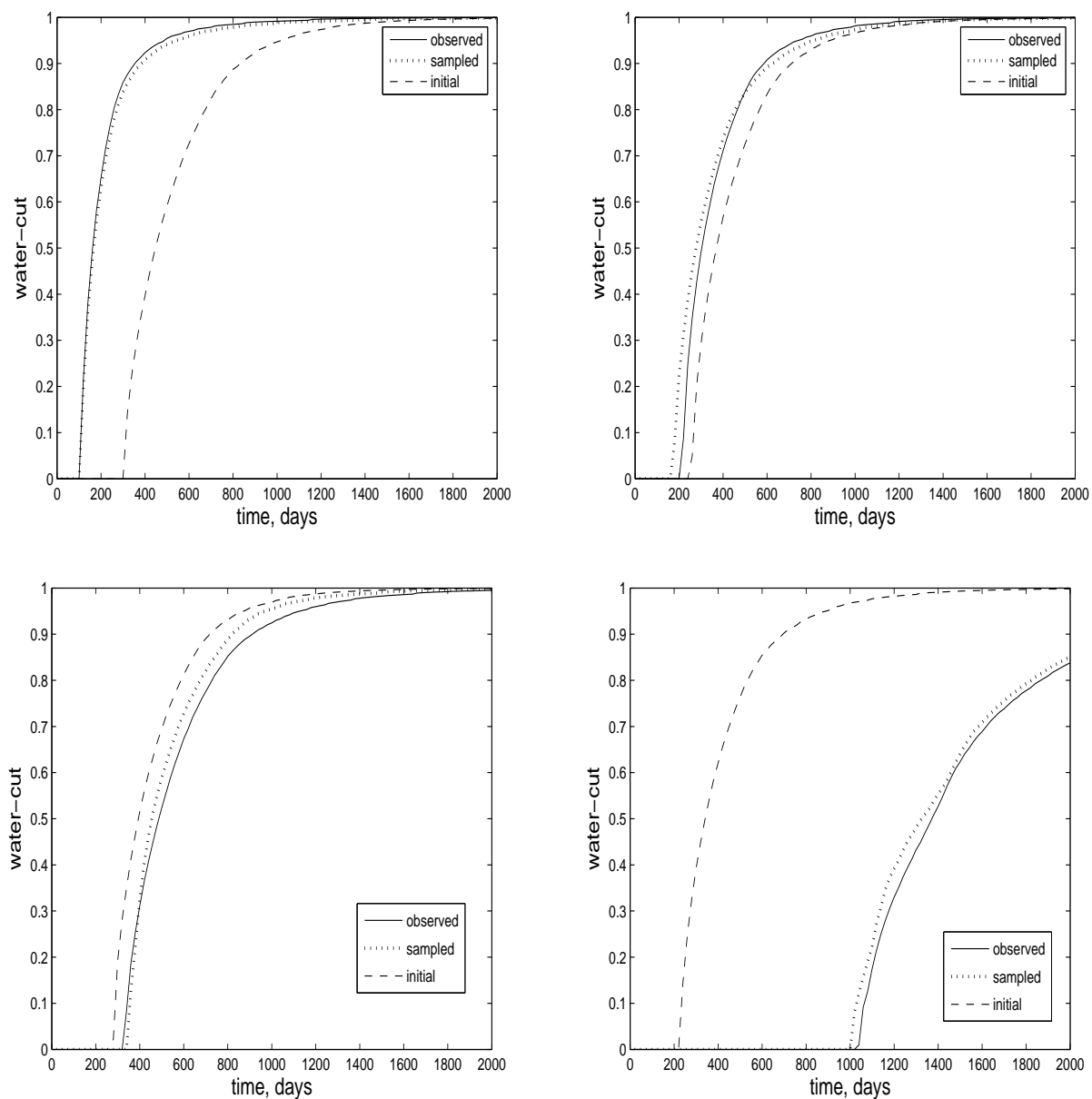

Figure 4. Initial and the final water-cut curves at production wells compared to the observed water-cut.

reference

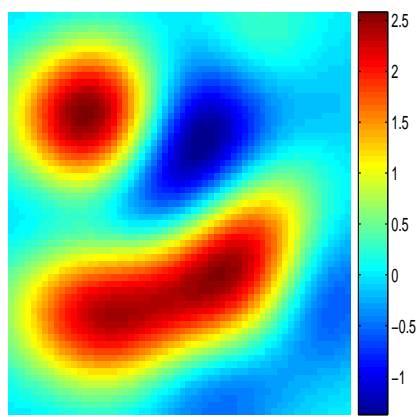

realization

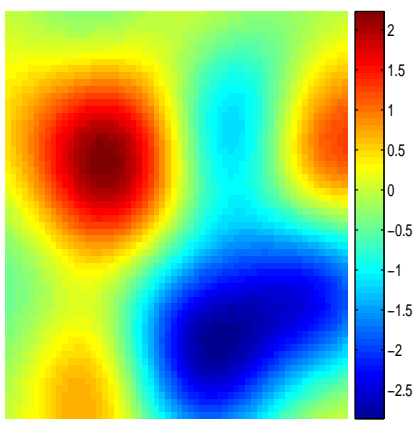

realization

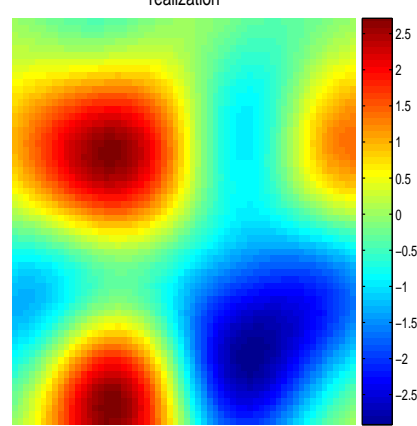

Figure 5. Realizations of the sampled permeability field. 